\title{
Visceral white fat remodelling contributes to intermittent hypoxia-induced atherogenesis
}

\author{
Laureline Poulain ${ }^{1,2}$, Amandine Thomas ${ }^{1,2}$, Jennifer Rieusset ${ }^{3}$, Louis Casteilla ${ }^{4}$, \\ Patrick Levy ${ }^{1,2,5}$, Claire Arnaud ${ }^{1,2,7}$ and Maurice Dematteis $1,2,6,7$
}

Affiliations: 'INSERM U1042, HP2 Laboratory, Grenoble, ${ }^{2}$ Faculté de Médecine, Université Joseph Fourier, Grenoble, ${ }^{3}$ INSERM UMR-1060, CarMeN Laboratory, Lyon, 4 UMR UPS/CNRS/EFS 5273 INSERM U1031, STROMALab, Toulouse, ${ }^{5}$ Laboratoires du Sommeil et EFCR, CHU, Hôpital A. Michallon, Grenoble, and ${ }^{6}$ Pôle Pluridisciplinaire de Médecine, CHU, Hôpital A. Michallon, Grenoble, France. ${ }^{7}$ Both authors contributed equally.

Correspondence: C. Arnaud, Laboratoire HP2, Institut Jean Roget, Faculté de Médecine de Grenoble, BP 170 , 38042 Grenoble Cedex 9, France. E-mail: claire.arnaudaujf-grenoble.fr

ABSTRACT Obstructive sleep apnoea is a highly prevalent disease characterised by repetitive upper airway collapse during sleep leading to intermittent hypoxia. Cardiometabolic complications of sleep apnoea have been mostly attributed to intermittent hypoxia. These consequences could be mediated through intermittent hypoxia-related alterations of the visceral white fat, as it is recognised for playing an important role in inflammation, atherogenesis and insulin resistance.

Epididymal adipose tissue alterations were investigated in 20-week-old nonobese male apolipoprotein Edeficient mice exposed to intermittent hypoxia (inspiratory oxygen fraction 5-21\%, 60-s cycle, $8 \mathrm{~h} \cdot$ day $^{-1}$ ) or air for 6 weeks. These adipose tissue alterations, as well as metabolic alterations and aortic atherosclerosis, were then assessed in lipectomised or sham-operated mice exposed to intermittent hypoxia or air for 6 weeks.

Intermittent hypoxia induced morphological (shrunken adipocytes), functional (increased uncoupling protein-1 expression) and inflammatory (increased macrophage recruitment and secretion of interleukin-6 and tumour necrosis factor- $\alpha$ ) remodelling of epididymal adipose tissue. Hypoxic mice presented more severe dyslipidaemia and atherosclerosis lesions and developed insulin resistance. Epididymal lipectomy attenuated both intermittent hypoxia-induced dyslipidaemia and atherogenesis, but did not improve insulin sensitivity.

Our results confirmed that the dyslipidaemic and proatherogenic effects of intermittent hypoxia are partly mediated through morphological, functional and inflammatory remodelling of visceral white fat, regardless of obesity.

@ERSpublications

Dyslipidaemic and proatherogenic effects of intermittent hypoxia are partly mediated by visceral white fat remodelling http://ow.ly/r3rLz

Received: Feb 012013 | Accepted after revision: June 192013 | First published online: Sept 262013

Support statement: This research was funded by a grant from AGIR@dom to M. Dematteis and a grant from the Heart and Arteries Foundation to C. Arnaud and M. Dematteis.

Conflict of interest: Disclosures can be found alongside the online version of this article at www.erj.ersjournals.com 


\section{Introduction}

Obstructive sleep apnoea (OSA) is a public health problem, as it affects $>10 \%$ of middle-aged males, and represents a main cause of cardiovascular morbidity and mortality [1]. Repetitive upper airway collapses during sleep result in intermittent hypoxia, thought to be responsible for cardiovascular complications such as atherosclerosis [2]. In OSA patients, increased carotid intima media thickness (IMT), an early sign of atherosclerosis, correlates with nocturnal oxygen desaturation, independently of other cardiovascular risk factors $[3,4]$. The rodent sleep apnoea model (i.e. rodents exposed to intermittent hypoxia) confirmed the detrimental role of hypoxia in OSA-associated vascular alterations; intermittent hypoxia increases aortic IMT in C57BL6 mice $[5,6]$ and accelerates the development of atherosclerotic lesions in C57BL6 mice on a high cholesterol diet [7] and in atherosclerosis-prone apolipoprotein E-deficient (ApoE ${ }^{-/-}$) mice $[8,9]$. However, the direct relationship between sleep apnoea or intermittent hypoxia and its cardiovascular consequences is not fully elucidated. We and others have evidence of a role for haemodynamic alterations [5], inflammation [6] and metabolic dysregulations [10, 11]. OSA is also frequently associated with obesity, which is the main confounder for detrimental consequences of OSA [12]. Indeed, obesity is an independent risk factor for diabetes and cardiovascular events, and is directly associated with increased mortality [13]. In addition to its function as the largest energy storage compartment of the body, adipose tissue has emerged as a hormonal organ, actively modulating metabolism and inflammation through the release of numerous cytokines and adipokines [14]. In obesity, excessive visceral white adipose tissue (WAT) contributes to metabolic dysregulation, vascular lesions and chronic inflammation through autocrine and paracrine regulations [15]. For example, WAT of obese mice exhibits increased tumour necrosis factor (TNF) $-\alpha$ expression and secretion, which is known to mediate insulin resistance [15]. In addition, a recent study showed that atherosclerosis-prone mice transplanted with inflamed epididymal WAT (EWAT) (the largest and easily harvested visceral WAT depot in mice) developed significantly more atherosclerosis, and that reduction in EWAT inflammation reduced atherosclerosis [16]. Therefore, inflammation of visceral WAT seems to be an important factor for vascular complications and may represent a link between obesity and vascular disease [15].

We have shown that intermittent hypoxia induced vascular and systemic inflammation in nonobese C57BL6 [6] and ApoE ${ }^{-/-}$[9] mice, and noted that intermittent hypoxia also induced inflammatory alterations and morphological changes of EWAT, with histological features of brown adipose tissue [17]. Therefore, we hypothesised that intermittent hypoxia itself could induce EWAT remodelling, which could contribute to metabolic and vascular disorders, independently of any obesity. The aims of the present study were firstly to characterise intermittent hypoxia-induced functional and structural alterations of EWAT using a nonobese mouse model of intermittent hypoxia, then to determine the contribution of this fat remodelling to intermittent hypoxia-induced metabolic and vascular alterations using EWAT-lipectomised animals.

\section{Methods}

Animals

35 20-week-old male ApoE ${ }^{-/-}$mice (C57BL6 background) fed on a standard-chow diet were used. They were weighed throughout the experiments, and food intake was measured daily. The study was conducted in accordance with the European Convention for the Protection of Vertebrate Animals used for Experimental and Other Scientific Purposes [18], and to the Guide for Care and Use of Laboratory Animals [19].

\section{Intermittent hypoxia}

Intermittent hypoxia was performed as previously described [6]. The animals were exposed during daytime $\left(\mathrm{n}=10\right.$ per cage, $8 \mathrm{~h} \cdot \mathrm{day}^{-1}$, cyclic inspiratory oxygen fraction $\left(\mathrm{FIO}_{2}\right) 5-21 \%, 60-\mathrm{s}$ cycle $\left(60 \mathrm{events}^{-1} \mathrm{~h}^{-1}\right)$ and lowest blood oxygen saturation up to 60\%) for 6 weeks. $\mathrm{FIO}_{2}$ was measured with a gas analyser (ML206, ADInstruments GmbH, Spechbach, Germany) throughout the experiment. Control animals (normoxic mice) were exposed to air in similar cages to reproduce similar noise and turbulences to those of the intermittent hypoxia stimulus. Ambient temperature was maintained at $20-22^{\circ} \mathrm{C}$.

\section{Adipose tissue alterations}

On the day following the last exposure period, animals were anesthetised by intraperitonal ketaminexylazine mixture $\left(100 \mathrm{mg} \cdot \mathrm{kg}^{-1} / 10 \mathrm{mg} \cdot \mathrm{kg}^{-1}\right)$ for bilateral EWAT collection. The tissue was either snap frozen in liquid nitrogen then stored at $-80^{\circ} \mathrm{C}$ for mRNA study, or fixed in $90 \%$ ethanol for immunohistochemistry or incubated for cytokine determinations.

\section{Immunohistochemical study}

Ethanol-fixed, paraffin-embedded EWAT were sectioned $(3.5 \mu \mathrm{m})$, deparaffinised in toluene and rehydrated in descending ethanol series, then stained with haematoxylin and eosin to assess tissue 
morphology. Adipocyte size was measured from photographs $(10 \times 40$ magnification $)$ using NIS-Elements microscope imaging software (Nikon Instruments Europe BV, Amsterdam, the Netherlands).

For macrophage staining, sections were incubated overnight at $4^{\circ} \mathrm{C}$ with a rat anti-F4/80 (1:10; eBiosciences SAS, Paris, France). Endogenous peroxidase activity was blocked with hydrogen peroxide. Nonspecific binding was blocked with normal goat serum (Vector Clinisciences SAS, Nanterre, France). Specific staining was detected using the N-Histofine Simple Stain Mouse Max-PO (Nichirei Biosciences Inc., Tokyo, Japan) followed by hematoxylin counterstaining. Macrophage infiltration was calculated as the ratio of nuclei of F4/80 positive cells to total nuclei counted in eight fields for each animal ( $n=5$ mice per group).

Uncoupling protein-1 and monocyte chemoattractant protein-1 mRNA expression

Uncoupling protein (UCP)-1, the hallmark of brown adipocytes, and monocyte chemoattractant protein (MCP)-1 mRNA levels were determined by reverse transcription followed by real-time quantitative reverse transcriptase (qRT)-PCR. Total mRNA was extracted from epididymal fat of intermittent hypoxic and normoxic mice ( $n=7-11$ per group), isolated with Tri-reagent (Invitrogen Life Technologies SAS, Saint Aubin, France) and analysed by qRT-PCR (ABI-7500; Applied Biosystems Life Technologies SAS, or LightCycler; Roche Diagnostics, Meylan, France). Each sample was analysed in duplicate, and 36B4 or TATA box-binding protein mRNA levels were simultaneously analysed as housekeeping genes (table 1).

Cytokine secretion

EWAT was isolated from mice and weighed. Each pad was divided into two equal pieces and incubated at $37^{\circ} \mathrm{C}$ with mild shaking in RPMI medium only (Invitrogen) or with the $\beta$-adrenergic agonist isoprenaline $\left(4 \times 10^{-5} \mathrm{M}\right.$; Hospira Enterprises BV, Almere, the Netherlands). The basal condition was defined as cytokine secretion in the absence of the lipolytic agent isoprenaline. After $120 \mathrm{~min}$ of incubation, interleukin (IL)- 6 and TNF- $\alpha$ were measured in the supernatants using ELISA, according to the manufacturer's instructions (R\&D Systems Europe, Lille, France). Cytokine concentrations were expressed as $\mathrm{ng} \cdot \mathrm{mL}^{-1}$ for $1 \mathrm{~g}$ of adipose tissue. Plasma levels of IL-6, TNF- $\alpha$ and adiponectin were measured using ELISA (R\&D Systems). Circulating leptin level was evaluated using MILLIPLEX MAP Mouse Adipokine Panel based on multiplex technology (Millipore SAS, Molsheim, France).

\section{The role of epididymal adipose tissue \\ Epididymal lipectomy}

In another series of mice, the animals were either lipectomised or sham-operated under intraperitoneal ketamine-xylazine $\left(100 \mathrm{mg} \cdot \mathrm{kg}^{-1} / 10 \mathrm{mg} \cdot \mathrm{kg}^{-1}\right)$ anaesthesia. Bilateral EWAT was carefully removed to keep the gonads intact. Sham animals underwent the same surgical protocol without removing the fat. Surgery sites were closed with 4-0 nylon absorbable monofilament (Ethicon SAS, Issy-Les-Moulineaux, France) and mice received buprenorphine $\left(0.1 \mathrm{mg} \cdot \mathrm{kg}^{-1}\right.$ i.p.). After 3 days during which body weight was measured daily, the animals were exposed to intermittent hypoxia or normoxia for 6 weeks, therefore constituting four experimental groups (lipectomised intermittent hypoxic and normoxic mice and sham intermittent hypoxic and normoxic mice, $n=7-10$ per group). On the day following the last exposure period, the animals were anaesthetised as described above. Blood was collected by cardiac puncture. The thoracoabdominal aorta, including the aortic roots, was removed and snap-frozen in liquid nitrogen and stored at $-80^{\circ} \mathrm{C}$ until analysis.

\section{Metabolic alterations}

Lipid measurements

Total and high-density lipoprotein cholesterol and triglycerides were measured in plasma by a colorimetric enzymatic reaction using the Modular P analyser (Roche Diagnostics). Low-density lipoprotein (LDL)cholesterol was calculated using the Friedewald formula.

\section{TABLE 1 Primers used for quantitative reverse transcriptase PCR analysis}

$\begin{array}{lll}\text { Gene } & \text { Forward primer sequence } \quad \text { Reverse primer sequence }\end{array}$

\begin{tabular}{lcc}
\hline UCP-1 & GAC-CGA-CGG-CCT-TTT-TCA-A & AAA-GCA-CAC-AAA-CAT-GAT-GAC-GTT \\
MCP-1 & TGG-AGC-ATC-CAC-GTG-TTG-GC & ACT-ACA-GCT-TCT-TTG-GGA-CA \\
TBP & AGT-CGG-AGG-AAT-CAG-ATG-AGG-AT & GGC-TGA-CTT-GGT-TGC-TTT-GG \\
36B4 & TGG-TGT-GCA-CAG-GAG-CCA-AG & TTC-ACA-TCA-CAG-CTC-CCC-AC
\end{tabular}

UCP: uncoupling protein; MCP: monocyte chemoattractant protein; TBP: TATA box-binding protein; 36B4: acidic ribosomal phosphoprotein (ribosomal protein, large, PO: RPLPO). 
Plasma levels of glycerol and nonesterified fatty acids (NEFA) were measured by colorimetric enzymatic reaction using the free glycerol reagent (Sigma-Aldrich, Lyon, France) and the NEFA-HR(2) (Wako Diagnostics, Neuss, Germany) assays, respectively.

Intraperitoneal insulin tolerance test

During intermittent hypoxia exposure, we used the intraperitoneal insulin tolerance test to assess global insulin sensitivity. Mice were fasted for $10 \mathrm{~h}$ then weighed before blood was collected from the tail tip for baseline glucose determination $(\mathrm{t}=0)$. Blood glucose was measured using the OneTouch Ultra glucometer (LifeScan France, Issy-Les-Moulineaux, France). Insulin $\left(0.75 \mathrm{IU} \cdot \mathrm{kg}^{-1}\right.$ body weight; Novo Nordisk A/S, Bagsvaerd, Denmark) was injected intraperitoneally, followed by further blood glucose measurements at 15 , 30, 60 and $90 \mathrm{~min}$ after the injection. The lowest blood glucose level (nadir) and its timing following insulin administration were calculated.

\section{Assessment of aortic lesions}

Atherosclerotic lesion size

Lesions of the thoracoabdominal aorta and aortic roots were analysed by Oil-red-O staining ( $\mathrm{n}=7-10$ per group). For each aortic root, we quantified lipid deposition from five sections ( $8 \mu \mathrm{m}$ thickness), separated from each other by $80 \mu \mathrm{m}$. Quantitative analysis of lipid deposition was performed using computer image analysis (MetaMorph 7 software, Zeiss Microscope; Molecular Devices France, St. Grégoire, France) after manual thresholding (thoraco-abdominal lesion) or contouring (aortic roots).

\section{Atherosclerotic lesion inflammation}

Macrophage and T-cell infiltration in the atherosclerotic lesions was assessed on immunostained aortic roots using antimouse macrophage (Mac-3, 1:100) and CD3 (1:1000; DakoCytomation, Les Ulis, France) antibodies. Anti- $\alpha$-smooth muscle actin antibody (1:50; Abcam, Paris, France) was used to stain smooth muscle cells. Nonspecific binding was blocked with normal goat serum (Vector). Primary antibodies were detected using the N-Histofine Simple Stain Mouse Max-PO (Nichirei Biosciences Inc.) followed by haematoxylin counterstaining. All stainings were assessed by computer-assisted image analysis (MetaMorph 7 software, Zeiss Microscope). Content of macrophages, smooth muscle cells and collagen were expressed as percentage of plaque size.

\section{Statistical analysis}

Results were expressed as mean \pm SEM. Differences between two groups over time were determined by twoway ANOVA for repeated measures, with a subsequent Tukey's post hoc test. For comparison between independent groups, t-tests or Mann-Whitney U-tests were used according to normality and variance homogeneity. Statistical significance was set at $\mathrm{p}<0.05$.

\section{Results}

Intermittent hypoxia induces morphological and functional remodelling of epididymal fat

EWAT from intermittent hypoxic mice exhibited shrunken adipocytes (fig. 1a) with increased expression of UCP-1 (fig. 1b) and thicker extracellular matrix (fig. 1c).

\section{Intermittent hypoxia induces inflammatory remodelling of epididymal fat}

The hypoxic WAT exhibited increased macrophage (F4/80-positive cells) infiltration (fig. 2a), organised in crown-like structures around individual adipocytes (fig. 2c) and increased expression of MCP-1 mRNA (fig. 2c). This inflammatory phenotype was confirmed by TNF- $\alpha$ and IL- 6 secretion from EWAT. At baseline, IL-6 and TNF- $\alpha$ were not modified by intermittent hypoxia (fig. $2 \mathrm{~d}$ and e). After isoprenaline stimulation, both cytokines increased in intermittent hypoxia animals, significantly for IL-6, but without reaching significance for TNF- $\alpha(\mathrm{p}=0.08)$. Whereas we observed localised inflammation in adipose tissue, we did not find evidence of systemic inflammation, as plasmatic cytokines (IL-6 and TNF- $\alpha$ ) were undetectable and adipokines (adiponectin and leptin) were not different between the four groups.

\section{Intermittent hypoxia induces insulin resistance and aggravates both dyslipidaemia and} atherosclerosis

The pre-existing dyslipidaemic pattern of the $\mathrm{ApoE}^{-/-}$mouse strain was aggravated by intermittent hypoxia, including higher plasma levels of total and LDL cholesterol, triglycerides, glycerol and NEFA in sham hypoxic compared to normoxic animals (table 2). Sham-operated hypoxic mice developed insulin resistance as shown by the lower glycaemia decline following insulin administration (fig. 3). Intermittent hypoxia also accelerated the development of atherosclerosis, as shown by the larger lesions in thoracoabdominal aorta and aortic roots (fig. 4). The atherosclerotic plaque composition was not affected 

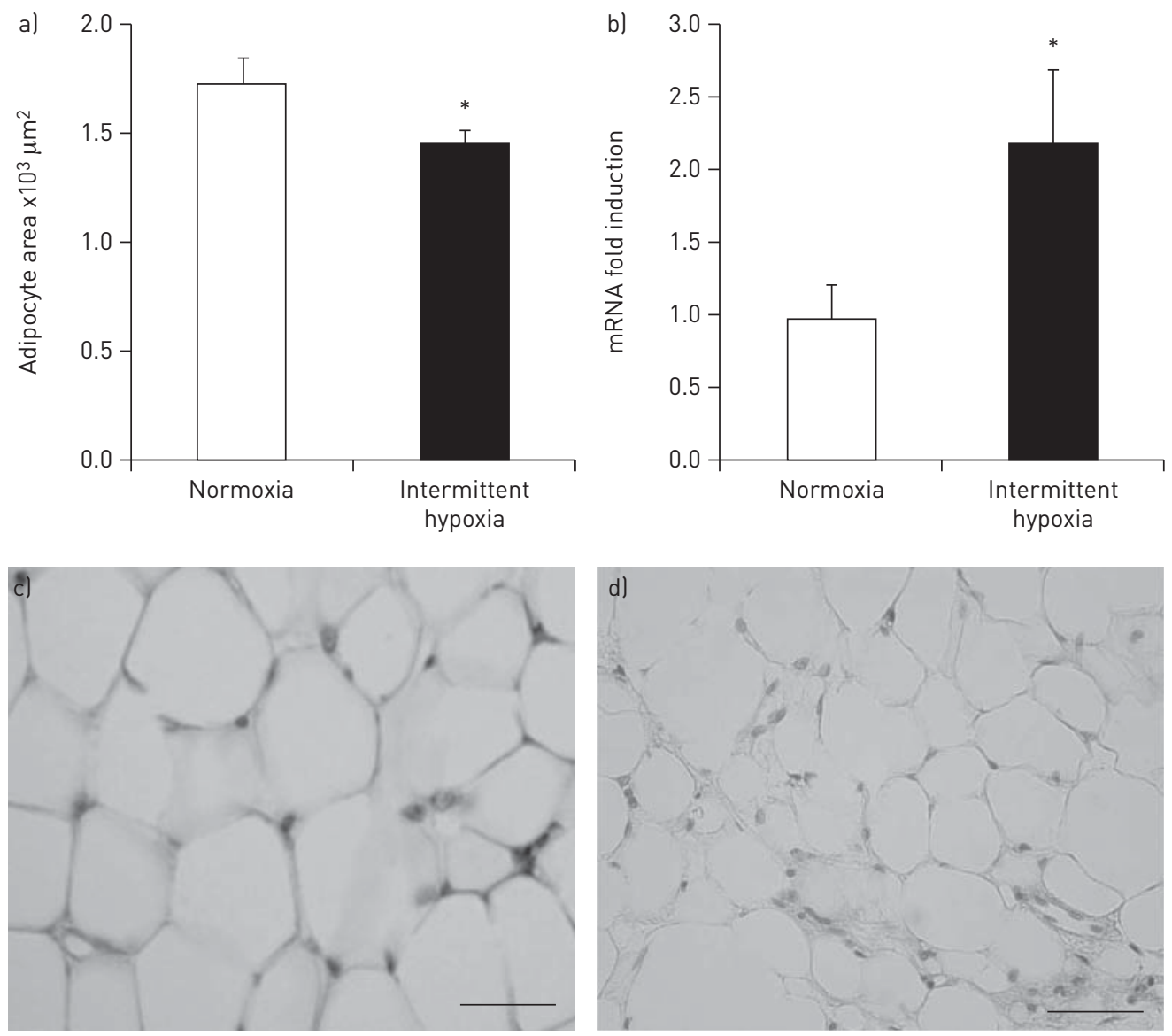

FIGURE 1 Intermittent hypoxia induces epididymal white adipose tissue morphological and functional remodelling. a) Quantification of adipocyte size after 6 weeks of intermittent hypoxia or normoxia. $n=7-8$ per group. ${ }^{*}: \mathrm{p}<0.05$ versus normoxia. b) Adipose tissue mRNA expression of uncoupling protein-1. Measurement is normalised to $36 \mathrm{~B} 4$ mRNA. $\mathrm{n}=7-8$ per group. ${ }^{*}: \mathrm{p}<0.05$ versus normal. $\mathrm{c}$ ) and d) Haematoxylin and eosin staining of adipose tissue from c) normoxic and d) intermittent hypoxic mice. Scale bar $=50 \mu \mathrm{m}$.

by intermittent hypoxia, as shown by the similar plaque content of macrophages (normoxia $5.40 \pm 1.40 \%$ versus intermittent hypoxia $3.81 \pm 0.56 \%$ ), T-cells (normoxia $0.25 \pm 0.05 \%$ versus intermittent hypoxia $0.29 \pm 0.04 \%$ ) and smooth muscle cells (normoxia $4.5 \pm 1.53 \%$ versus intermittent hypoxia $4.16 \pm 1.20 \%$ ).

\section{Epididymal lipectomy prevents intermittent hypoxia-induced dyslipidaemia and atherosclerosis} Lipectomy prevented the intermittent hypoxia-related disturbances in plasma cholesterol, whereas glycerol and NEFA levels remained similar to those of sham hypoxic animals (table 2). The proatherogenic effect of intermittent hypoxia was also prevented by lipectomy, as atherosclerotic plaque size in the thoracoabdominal aorta was very similar to those of normoxic animals (fig. $4 \mathrm{a}$ and b). This effect was more modest in the aortic roots (fig. $4 \mathrm{c}$ and d). In contrast, plaque quality was not modified by lipectomy, as shown by the plaque contents of macrophages (normoxia $3.02 \pm 0.62 \%$ versus intermittent hypoxia $3.99 \pm 0.66 \%$ ), T-cells (normoxia $0.29 \pm 0.07 \%$ versus intermittent hypoxia $0.26 \pm 0.05 \%$ ) and smooth muscle cells (normoxia $2.83 \pm 1.10$ versus intermittent hypoxia $5.0 \pm 1.96 \%$ ).

Regarding glucidic metabolism, lipectomy did not prevent the intermittent hypoxia-induced insulin resistance. In contrast, and in agreement with the buffering role of WAT in maintenance of glucose homeostasis, lipectomy induced insulin resistance in normoxic animals (fig. 3).

\section{Discussion}

The pathophysiology of OSA-induced atherogenesis and metabolic alterations remains poorly understood. Here, we showed in nonobese ApoE-deficient mice that 6 weeks of intermittent hypoxia led to morphological, functional and inflammatory remodelling of the visceral WAT, and this remodelling contributed to intermittent hypoxia-induced dyslipidaemia and atherosclerosis. This study is the first to 

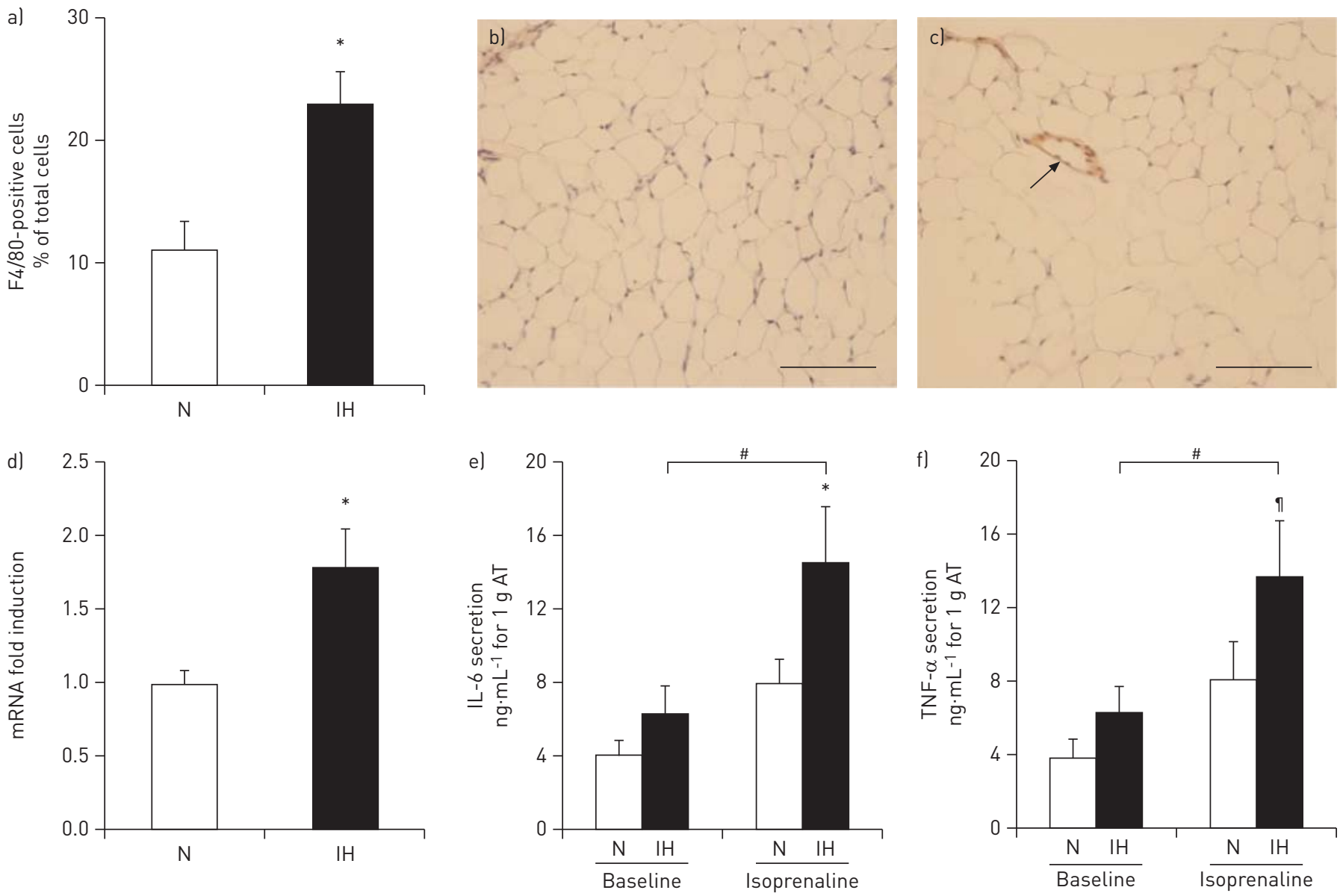

FIGURE 2 Intermittent hypoxia (IH) induces epididymal white adipose tissue inflammatory remodelling. a) Quantification of macrophage infiltration after 6 weeks of IH or normoxia (N). $n=5$ per group. ${ }^{*}$ : $\mathrm{p}<0.05$ versus normoxia. b) and c) Representative F4/80 immunostaining showing macrophages organised in a crown-like structure around adipocytes (arrow). Scale bars $=100 \mu \mathrm{m}$. d) Adipose tissue mRNA expression of monocyte chemoattractant protein-1. Measurement is normalised to TATA box-binding protein mRNA. $n=8-11$ per group. ${ }^{*}: \mathrm{p}<0.05$ versus normoxia. e) Interleukin (IL)- 6 and $\mathrm{f}$ ) tumour necrosis factor (TNF)- $\alpha$ secretion from epididymal adipose tissue (AT) at basal condition or after isoprenaline stimulation. $\mathrm{n}=13$ per group. ${ }^{*}: \mathrm{p}<0.05$ versus normoxia; ${ }^{*}$ : $\mathrm{p}<0.05$ versus basal condition; ${ }^{\circ}: \mathrm{p}=0.083$.

provide evidence that a physiological amount of visceral WAT exposed to intermittent hypoxia becomes pathological, behaving like excess fat in obesity.

\section{Methodological considerations}

The intermittent-hypoxia experimental model was developed over two decades ago and reproduces one of the major components of OSA, which is considered to be the most detrimental factor contributing to sleep apnoea complications [2]. However, in the absence of additional factors (obesity, high fat diet, etc) intermittent hypoxia needs to be severe enough to induce measurable and reproducible detrimental effects [2]. Therefore, and as previously published by our group and others, the hypoxic stimulus used in this study mimics severe sleep apnoea $[2,5,6]$.

\section{Intermittent hypoxia induces EWAT remodelling}

In lean mice, intermittent hypoxia induced moderate EWAT inflammation, including macrophage infiltration, increased MCP-1 expression and proinflammatory cytokine release (IL-6 and TNF- $\alpha$ ). Fat inflammation is well known in obesity and results at least in part from hypoxia, as, with fat expansion, adipocytes become too distant from blood vessels [20]. Through the repetitive hypoxia-reoxygenation sequence, intermittent hypoxia generates free radical stress and inflammation. Intermittent hypoxia also increases sympathoadrenergic activity, which in turn exacerbates oxidative stress [21], and the adipose expression of MCP-1 [22], which is a key chemokine regulating macrophage tissue infiltration [23]. We used isoprenaline to mimic the sympathetic overactivity occuring in OSA patients during sleep, which resulted in enhanced proinflammatory cytokine release. Collectively, our data suggest chronic low-grade EWAT inflammation with intermittent exacerbation during sympathetic activation. 
TABLE 2 Weight and biological characteristics in lipectomised and sham-operated apolipoprotein-E deficient mice exposed to intermittent hypoxia or normoxia

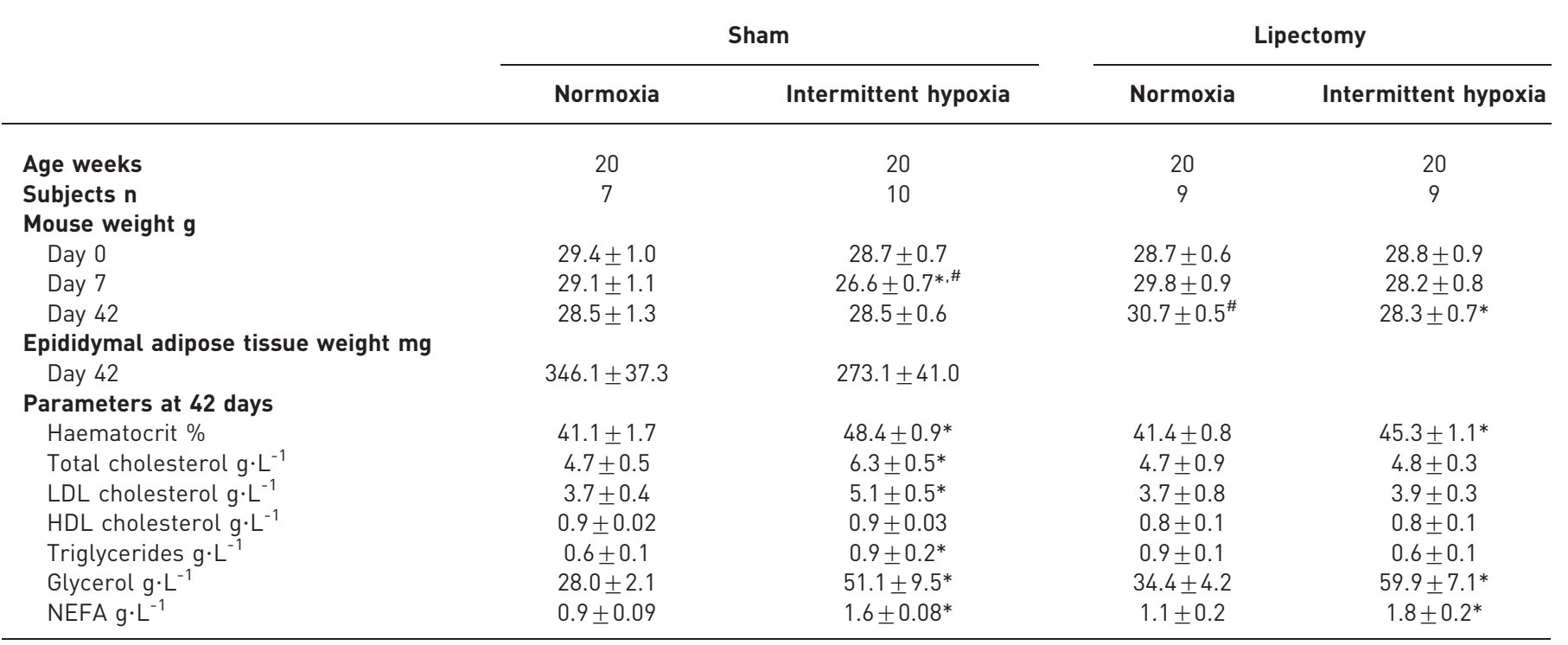

Data are presented as mean \pm SEM, unless otherwise stated. LDL: low-density lipoprotein; HDL: high-density lipoprotein; NEFA: nonesterified fatty acids. *: $p<0.05$ versus normoxia; ${ }^{\#}: \mathrm{p}<0.05$ versus day 0 .

a)

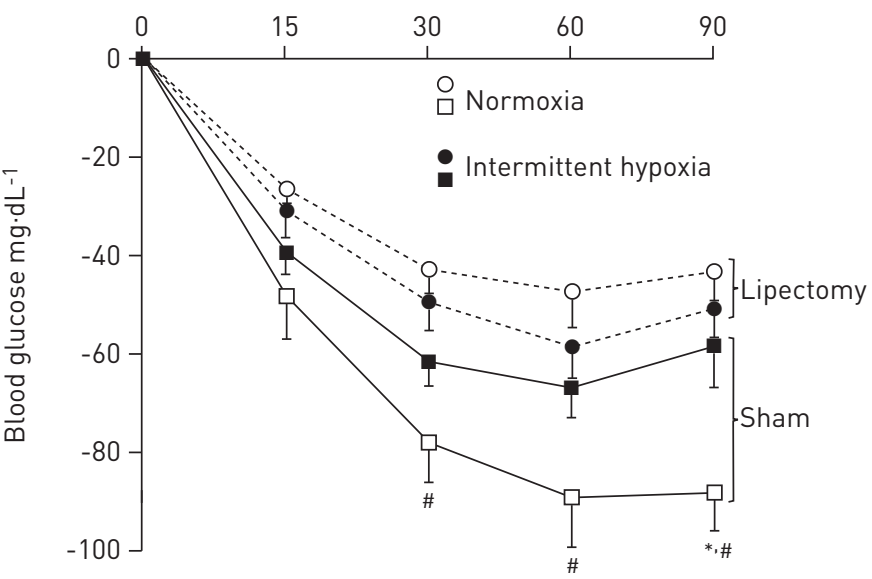

b)

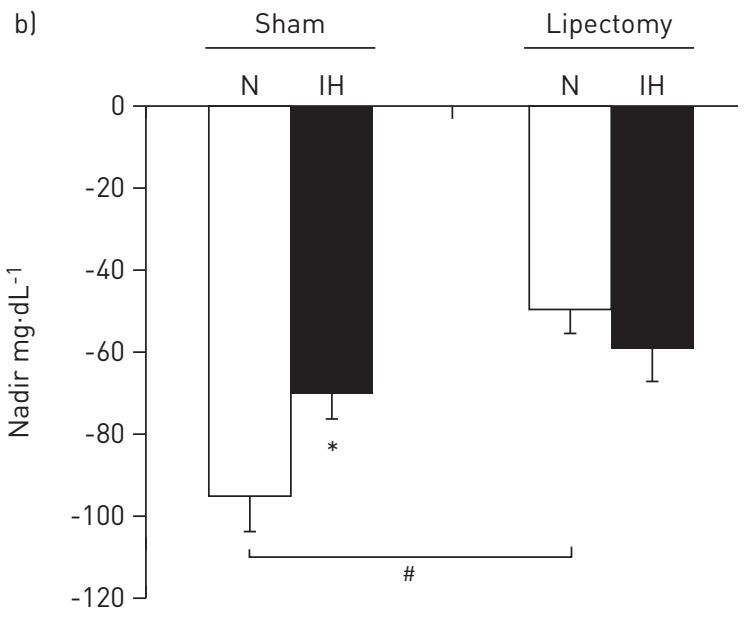

FIGURE 3 Intermittent hypoxia (IH) induces insulin resistance. a) Intraperitoneal insulin tolerance test in epididymal lipectomised or sham-operated mice exposed to IH or normoxia $(\mathrm{N})$ for 6 weeks; b) Lowest blood glucose level during the first 90 min (nadir). $\mathrm{n}=6-15$ per group. ${ }^{*}$ : $\mathrm{p}<0.05$ versus $\mathrm{N}$; ${ }^{*}$ : $\mathrm{p}<0.05$ versus sham $\mathrm{N}$. 


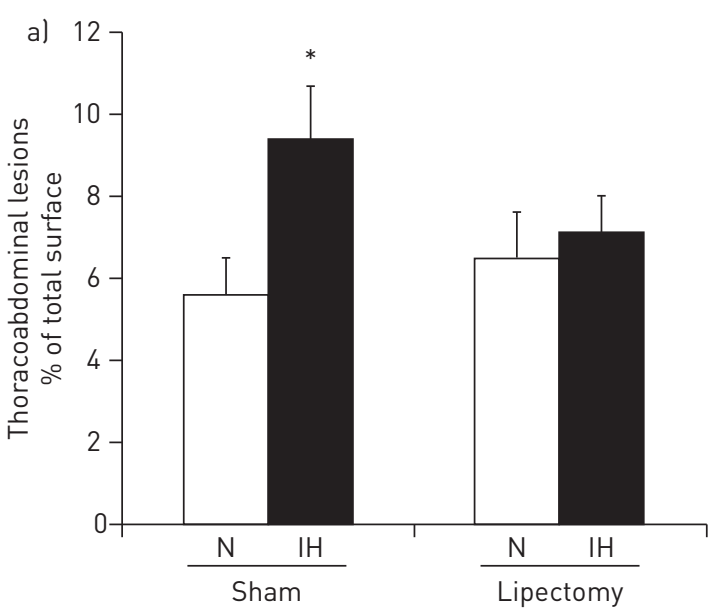

b)
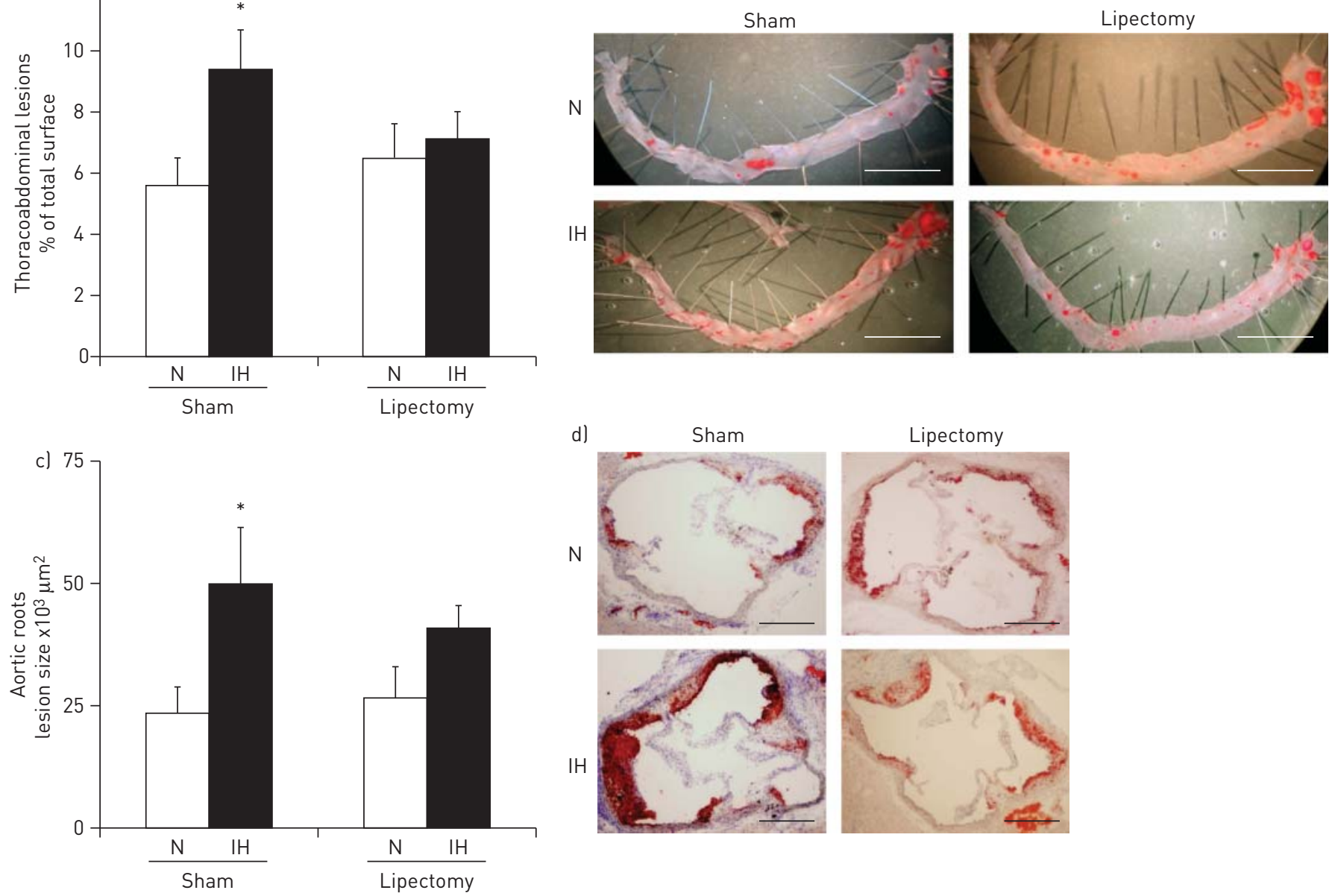

d)

Sham

N

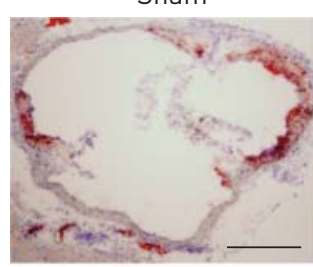

$\mathrm{IH}$

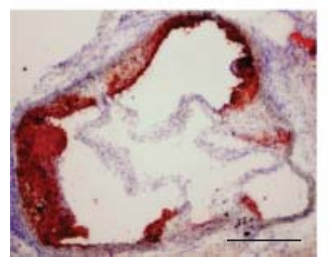

Lipectomy

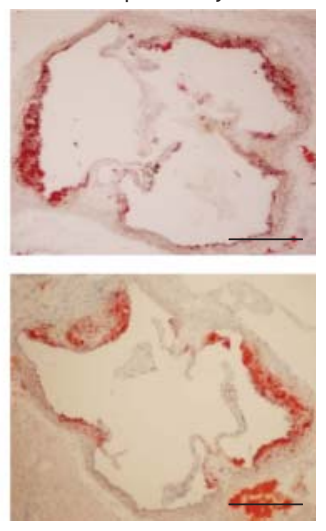

FIGURE 4 Epididymal lipectomy attenuates intermittent hypoxia (IH)-induced atherogenesis. a) Thoracoabdominal aortic lesions expressed as percentage of total aorta surface in lipectomised or sham-operated mice exposed to IH or normoxia (N) for 6 weeks. b) Representative photographs of thoracoabdominal lesions. Scale bars $=0.5 \mathrm{~cm}$. c) Size of atherosclerotic lesions in aortic roots expressed in $\mu \mathrm{m}^{2} . \mathrm{d}$ ) Representative photographs of aortic root lesions. Scale bars $=1000 \mu \mathrm{m} . \mathrm{n}=10$ per group. ${ }^{*}: \mathrm{p}<0.05$ versus $\mathrm{N}$.

\section{Intermittent hypoxia induces insulin resistance, dyslipidaemia and atherosclerosis}

As previously, we used ApoE-deficient mice to assess the atherogenic and metabolic effects of intermittent hypoxia, as they are atherosclerosis-prone animals and develop dyslipidaemia and insulin resistance. We confirmed the atherogenic and dyslipidaemic effects of intermittent hypoxia; hypoxic mice exhibited larger aortic atherosclerotic lesions and higher plasmatic levels of cholesterol (total and LDL) and triglycerides. Lipid alterations, constituting an early step of atherogenesis [25], have been previously described both in OSA patients and in mice exposed to intermittent hypoxia [26]. Intermittent hypoxia also increased plasmatic glycerol and NEFA, and aggravated the insulin resistance. WAT constitutes the main source of NEFA, also called free fatty acids (FFAs), and excessive circulating FFAs contribute to insulin resistance [27, 28]. The relationship between fat inflammation, in particular macrophage-related inflammation in WAT, and insulin resistance is now largely described in the literature [14]. Our results are in agreement with clinical studies showing a progressive worsening of insulin resistance and metabolic syndrome with OSA severity [26]. Exposure to intermittent hypoxia also leads to decreased insulin sensitivity in healthy volunteers [29] and in lean mice [30]. Collectively, our data and those from the literature demonstrate that intermittent hypoxia induces metabolic disorders independently of any obesity, and suggest that intermittent hypoxia-induced adipose tissue alterations could represent one of the links between inflammation, insulin resistance and vascular consequences.

\section{EWAT lipectomy attenuates the dyslipidaemic and atherogenic effects of intermittent hypoxia}

We performed bilateral EWAT lipectomy before exposure to assess the specific role of EWAT in intermittent hypoxia-induced cardiovascular and metabolic alterations. Lipectomised animals had smaller atherosclerotic lesions in the thoracoabdominal aorta, whereas the preventive effect was less in the aortic root. 
This suggests that, beyond WAT alterations, the atherogenic effect of intermittent hypoxia involves additional mechanisms such as regional aortic susceptibility to develop atherosclerosis, e.g. aortic roots are prone to develop the largest lesions in particular due to haemodynamic factors.

From our results, the lesser atherosclerosis progression after lipectomy appears to be related to the prevention of intermittent hypoxia-induced dyslipidaemia, as lipectomised hypoxic mice had similar levels of total and LDL cholesterol to normoxic animals. However, the beneficial effect of lipectomy may also be related to the prevention of EWAT inflammation. We did not investigate the mechanisms underlying the beneficial effect on lipid levels as it was beyond the scope of this study. Because we found inflammatory alterations in EWAT, we chose to focus on proinflammatory cytokines, but other signalling proteins, such as the adipose angiopoietin-like 4, a potent lipoprotein lipase inhibitor, are probably involved in intermittent hypoxia-associated atherosclerosis and may have contributed to the preventive effect of lipectomy [31]. In contrast, the intermittent hypoxia-induced elevation in plasma glycerol and FFAs remained unchanged. Lipectomy in this study was limited to the epididymal compartment, which is the largest and most easily harvested visceral WAT depot in mice. However, there are other WAT territories such as mesenteric, perirenal and subcutaneous WAT. Glycerol and FFAs are two products of lipolysis occurring in the various adipose tissues, including epididymal and subcutaneous tissues. Although visceral WAT is predominantly associated with metabolic disease, the majority of circulating FFAs originate from subcutaneous WAT [32].

Regarding insulin sensitivity, whereas it has been shown that removing visceral fat could prevent insulin resistance in obese or ageing rats [33], we found that EWAT lipectomy induced insulin resistance in lean normoxic animals. These data confirm the major role of WAT in the homeostatic regulation of glucose; it is indeed one of the primary targets of insulin-stimulated glucose uptake, and it regulates insulin sensitivity through the release of numerous cytokines and adipokines. Therefore clinical situations with quantitative (excessive or overly reduced adipose mass) and/or qualitative alterations of the WAT may lead to insulin resistance [34]. We found that sham-operated hypoxic mice and lipectomised normoxic mice exhibited similar insulin resistance, suggesting that EWAT in animals exposed to intermittent hypoxia was functionally impaired for glucose buffering. As a consequence, lipectomy in hypoxic animals did not further aggravate the insulin resistance.

\section{Conclusion}

Although the causative mechanisms remain to be elucidated, we showed that intermittent hypoxia had detrimental effects on EWAT of nonobese mice, and these contributed to the cardiovascular and metabolic complications induced by intermittent hypoxia. EWAT exhibited inflammatory alterations that were exacerbated under $\beta$-adrenergic activation, the latter mimicking the sympathetic activation induced by sleep apnoea events. Therefore, our original findings suggest novel therapeutic options for OSA patients, as compliance with the gold standard continuous positive airway pressure therapy is limited, and losing fat through weight reduction is applicable only in obese OSA patients. Targeting inflammation, either using drugs or by improving lifestyle (diet and exercise), and buffering nocturnal sympathetic activation could provide further therapeutic options for OSA patients.

\section{References}

1 Somers VK, White DP, Amin R, et al. Sleep apnea and cardiovascular disease: an American Heart Association/ American College of Cardiology Foundation Scientific Statement from the American Heart Association Council for High Blood Pressure Research Professional Education Committee, Council on Clinical Cardiology, Stroke Council, and Council On Cardiovascular Nursing. Circulation 2008; 118: 1080-1111.

2 Dematteis M, Godin-Ribuot D, Arnaud C, et al. Cardiovascular consequences of sleep-disordered breathing: contribution of animal models to understanding the human disease. ILAR J 2009; 50: 262-281.

3 Baguet JP, Hammer L, Lévy P, et al. The severity of oxygen desaturation is predictive of carotid wall thickening and plaque occurrence. Chest 2005; 128: 3407-3412.

4 Drager LF, Bortolotto LA, Lorenzi MC, et al. Early signs of atherosclerosis in obstructive sleep apnea. Am J Respir Crit Care Med 2005; 172: 613-618.

5 Dematteis M, Julien C, Guillermet C, et al. Intermittent hypoxia induces early functional cardiovascular remodeling in mice. Am J Respir Crit Care Med 2008; 177: 227-235.

6 Arnaud C, Beguin PC, Lantuejoul S, et al. The inflammatory preatherosclerotic remodeling induced by intermittent hypoxia is attenuated by RANTES/CCL5 inhibition. Am J Respir Crit Care Med 2011; 184: 724-731.

7 Savransky V, Nanayakkara A, Li J, et al. Chronic intermittent hypoxia induces atherosclerosis. Am J Respir Crit Care Med 2007; 175: 1290-1297.

8 Jun J, Reinke C, Bedja D, et al. Effect of intermittent hypoxia on atherosclerosis in apolipoprotein E-deficient mice. Atherosclerosis 2010; 209: 381-386.

9 Arnaud C, Poulain L, Lévy P, et al. Inflammation contributes to the atherogenic role of intermittent hypoxia in apolipoprotein-E knock out mice. Atherosclerosis 2011; 219: 425-431.

10 Li J, Thorne LN, Punjabi NM, et al. Intermittent hypoxia induces hyperlipidemia in lean mice. Circ Res 2005; 97: 698-706. 
11 Li J, Nanayakkara A, Jun J, et al. Effect of deficiency in SREBP cleavage-activating protein on lipid metabolism during intermittent hypoxia. Physiol Genomics 2007; 31: 273-280.

12 Bonsignore MR, McNicholas WT, Montserrat JM, et al. The adipose tissue in obesity and obstructive sleep apnoea. Eur Respir J 2012; 39: 746-767.

13 Bigaard J, Frederiksen K, Tiønneland A, et al. Waist circumference and body composition in relation to all-cause mortality in middle-aged men and women. Int J Obes (Lond) 2005; 29: 778-784.

14 Olefsky JM, Glass CK. Macrophages, inflammation, and insulin resistance. Annu Rev Physiol 2010; 72: 219-246. Shoelson SE, Lee J, Goldfine AB. Inflammation and insulin resistance. J Clin Invest 2006; 116: 1793-1801.

16 Ohman MK, Shen Y, Obimba CI, et al. Visceral adipose tissue inflammation accelerates atherosclerosis in apolipoprotein E-deficient mice. Circulation 2008; 117: 798-805.

17 Poulain L, Rieusset J, Levy PA, et al. Intermittent hypoxia induces dyslipidemia and atherogenesis through visceral white adipose tissue inflammation. Am J Respir Crit Care Med 2012; 185: A3867.

18 Council of Europe. European Convention for the Protection of Vertebrate Animals used for Experimental and Other Scientific Purposes. European Treaties ETS 123. Strasbourg, March 18, 1986.

19 National Research Council. Guide for the Care and Use of Laboratory Animals. Washington, National Academies Press, 1996.

20 Trayhurn P, Wang B, Wood IS. Hypoxia in adipose tissue: a basis for the dysregulation of tissue function in obesity? Br J Nutr 2008; 100: 227-235.

21 Prabhakar NR, Kumar GK, Peng YJ. Sympatho-adrenal activation by chronic intermittent hypoxia. J Appl Physiol 2012; 113: 1304-1310.

22 Wang YY, Lin SY, Chuang YH, et al. Adipose proinflammatory cytokine expression through sympathetic system is associated with hyperglycemia and insulin resistance in a rat ischemic stroke model. Am J Physiol Endocrinol Metab 2010; 300: E155-E163.

23 Kanda H, Tateya S, Tamori Y, et al. MCP-1 contributes to macrophage infiltration into adipose tissue, insulin resistance, and hepatic steatosis in obesity. J Clin Invest 2006; 116: 1494-1505.

24 Smorlesi A, Frontini A, Giordano A, et al. The adipose organ: white-brown adipocyte plasticity and metabolic inflammation. Obes Rev 2012; 13: Suppl. 2, 83-96.

25 Hansson GK. Inflammation, atherosclerosis, and coronary artery disease. N Engl J Med 2005; 352: 1685-1695.

Jun J, Polotsky VY. Metabolic consequences of sleep-disordered breathing. ILAR J 2009; 50: 289-306.

Bays H, Mandarino L, DeFronzo RA. Role of the adipocyte, free fatty acids, and ectopic fat in pathogenesis of type 2 diabetes mellitus: peroxisomal proliferator-activated receptor agonists provide a rational therapeutic approach. J Clin Endocrinol Metab 2004; 89: 463-478.

28 Jensen MD. Is visceral fat involved in the pathogenesis of the metabolic syndrome? Human model. Obesity (Silver Spring) 2006; 14: Suppl. 1, 20S-24S.

29 Louis M, Punjabi NM. Effects of acute intermittent hypoxia on glucose metabolism in awake healthy volunteers. J Appl Physiol 2009; 106: 1538-1544.

30 Iiyori N, Alonso LC, Li J, et al. Intermittent hypoxia causes insulin resistance in lean mice independent of autonomic activity. Am J Respir Crit Care Med 2007; 175: 851-857.

31 Drager LF, Yao Q, Hernandez KL, et al. Chronic intermittent hypoxia induces atherosclerosis via activation of adipose angiopoietin-like 4. Am J Respir Crit Care Med 2013; 188: 240-248.

32 Klein S. The case of visceral fat: argument for the defense. J Clin Invest 2004; 113: 1530-1532.

33 Gabriely I, Ma XH, Yang XM, et al. Removal of visceral fat prevents insulin resistance and glucose intolerance of aging: an adipokine-mediated process? Diabetes 2002; 51: 2951-2958.

34 McKnight SL. WAT-free mice: diabetes without obesity. Genes Dev 1998; 12: 3145-3148. 